\title{
The History of Pertussis (Whooping Cough); 1906-2015: Facts, Myths, and Misconceptions
}

\author{
James D. Cherry ${ }^{1}$
}

Published online: 9 April 2015

(C) Springer International Publishing AG 2015

\begin{abstract}
Pertussis and Bordetella pertussis infections since 1906 are reviewed with an attempt to address facts, myths, and misconceptions. Clinical pertussis is a noninflammatory illness which occurs without fever or productive cough, and its hallmark is the distinctive paroxysmal cough. Because of its significant mortality and morbidity, whole-cell vaccines were developed in the 1930s, and the universal use of these vaccines decreased the incidence of reported pertussis 157-fold by 1970 . Because of real and perceived reactions to diphtheria and tetanus toxoids and whole cell pertussis vaccine (DTwP) vaccines, diphtheria and tetanus toxoids and acellular pertussis vaccine (DTaP) vaccines were developed and put into universal use in the USA in 1997. Pertussis has two epidemiologies; one is reported pertussis and the other is $B$. pertussis illness. The study of these two epidemiologies indicate that reported pertussis is just the tip of the iceberg and B. pertussis cough illnesses occur in all age groups and they are very common; also, asymptomatic infection is more common than symptomatic infection. DTaP vaccines are less reactogenic than DTwP vaccines, but their effectiveness is less. B. pertussis infection is caused by many protein antigens, but illness due to this organism is caused by just two factors, pertussis toxin (PT) and a "cough toxin" which, as yet, has not been discovered. The present "resurgence of pertussis" is mainly due to greater awareness and the use of PCR for diagnosis. There are also many other factors which
\end{abstract}

This article is part of the Topical Collection on Infectious Disease Epidemiology

James D. Cherry

jcherry@mednet.ucla.edu

1 Department of Pediatrics, David Geffen School of Medicine at UCLA, 10833 Le Conte Avenue, MDCC 22-442, Los Angeles, CA 90095-1972, USA have contributed to the "resurgence." New vaccines are clearly needed; with our present vaccines (DTaP and adolescent and adult formulated tetanus and diphtheria toxoids and acellular pertussis vaccine (Tdap)), if used correctly, severe pertussis and deaths in infants can be prevented.

Keywords Whooping cough $\cdot$ Pertussis $\cdot$ Bordetella pertussis $\cdot \mathrm{DTaP} \cdot \mathrm{DTwP} \cdot \mathrm{Tdap}$

\section{Introduction}

Between 1976 and 1979, I participated in a large study of diphtheria, tetanus, and pertussis (DTP) vaccine reactions [1]. During the ensuing 36 years, my research focused on pertussis epidemiology, diagnosis, vaccines, and

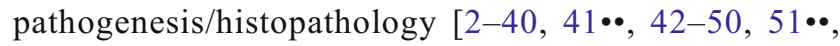
$52-54,55 \cdot 0,56]$. I have had the opportunity to examine the past as well as the present regarding pertussis and its causative agent Bordetella pertussis.

Compared with other contagious important infectious diseases like smallpox, polio, and measles, pertussis has no ancient history [57••, 58]. Pertussis is also distinct in that of all severe bacterial infections, it occurs without fever and other signs of inflammation unless there is a secondary bacterial or viral infection $[37,51 \bullet \bullet, 55 \bullet, 57 \bullet \bullet]$. The cause of epidemic pertussis, B. pertussis, was discovered in 1906 [59]. Over the last $\sim 100$ years, pertussis epidemiology has been extensively studied as has the microbiology of $B$. pertussis $[2,4,9-40$, $41 \bullet \bullet, 42-50,51 \bullet, 52-54,55 \bullet \bullet, 56,57 \bullet \bullet, 58,59,60 \bullet \bullet, 61-90]$. During this time, much has been learned, but many "facts" are wrong. In this communication, I will review numerous facts and fictions relating to the pathophysiology of $B$. pertussis infection, the epidemiology of infection and disease, and the present resurgence of pertussis. 


\section{Background}

\section{History}

Although there are scattered bits of evidence suggesting a longer history of pertussis, the first recognized epidemic of pertussis occurred in Paris, France in 1578 [3, 43, 58]. This was described by Guillaume de Baillou (Ballonius), and he gave a clear description of the illness [3].

In 1940, Holmes in his textbook described many of the peculiar features of pertussis (Table 1) [57••]. Unfortunately, Holmes' observations have been largely overlooked by pertussis microbiologists and clinical experts in the modern era. Also largely overlooked today are the seminal publications of the first half of the twentieth century by Mallory and Horner, Lapin, Holt, Mannerstedt, and many other keen observers [23, $57 \bullet \bullet, 58,60 \bullet \bullet, 61,62]$.

\section{Microbiology}

B. pertussis (the cause of epidemic whooping cough) is a fastidious gram-negative coccobacillus [37]. It was first isolated in 1906 by Jules Bordet and Octave Gengou [59]. The media that they developed (BG agar) is still commonly used in research and clinical laboratories today [37].

Since the morbidity and mortality due to $B$. pertussis infection in the early years of the twentieth century were staggering, the organism was the focus of major research efforts [3, 37]. In 1909, Bordet and Gengou, in studies in mice, described a toxin that was called dermonecrotic toxin (DNT) [37, 63]. Over the ensuing $\sim 100$ years, $B$. pertussis has been studied extensively by researchers in many countries. Numerous toxins and other virulence factors have been described [37]. In 2003, the complete genome of B. pertussis as well as two other Bordetella species was sequenced [64]. B. pertussis has a genome size of $4.09 \mathrm{Mb}$ and it contains $\sim 3436$ proteins.

Over the years, many toxins and virulence factors of $B$. pertussis have been found and studied in animal model systems, most commonly the mouse [37]. The various discoveries led to the development of acellular vaccines in the 1980s. Although the mouse model has led to numerous discoveries, it

Table 1 A summary of peculiar features of whooping cough
1. No ancient history
2. No characteristic changes
3. The paroxysmal cough is distinctive but we do not know why
4. It kills more girl babies than boys
5. Although it is an infectious disease, there is no fever during the spasmodic stage and no physical findings

From $[57 \bullet \cdot]$ is important to note that the illness in the mouse does not reflect that which occurs in humans $[53,55 \bullet \cdot]$. Studies in mice have led to much misinformation relating to pertussis and its prevention. The recently developed baboon model has already clarified some areas relating to $B$. pertussis infection [65-67]. However, whether the pathology in the baboon is the same as that in the human infant has not yet been determined.

\section{Clinical Pertussis}

Clinical pertussis was well described over 100 years ago [23, 61]. However, what was described was primary infection in young children. Except for the observations of a few clinicians, reinfection illnesses in adolescent and adult pertussis were rarely reported. Also, the picture was confused by concomitant or secondary viral and bacterial infections. For example fever, wheezing, or a truly productive cough do not occur in pertussis $[37,48,91]$. Interestingly, a recent paper from Southern Kaiser suggested that $8 \%$ of patients with pertussis had wheezing [68]. Since this is not true (unless there is a concomitant respiratory viral infection), why did this record review find the result that they reported? The probable answer to this relates to electronic records. In subacute cough illnesses, the physicians would like to use drugs like albuterol. To obtain the medication, they list wheezing in their history or physical examination. (This information was suggested to me by a Kaiser pediatric infectious diseases physician).

The clinical manifestations of $B$. pertussis infection are affected by many factors. Most important in this regard is whether the infection is occurring in a person who has been previously vaccinated or who had a previous infection, Tables 2 and 3.

\section{Pertussis Vaccines}

\section{History (Table 4)}

During the period 1926-1930, there were 36,013 deaths from pertussis in the USA; the highest number occurred in young infants $[2,3,58]$. Because of this mortality and significant

Table 2 Pertussis in young infants

- Initially infant looks deceptively well; coryza, sneezing, clearing throat, no fever, mild cough

- Paroxysmal stage: gagging, gasping, eye bulging, bradycardia, cyanosis, vomiting

- Leukocytosis with lymphocytosis

- Apneic episodes

- Seizures

- Respiratory distress

- Pneumonia

- Adenovirus or RSV coinfection can confuse picture 
Table 3 Clues in the clinical Dx of pertussis in older children, adolescents, and adults

- Lack of fever

- Lack of a truly productive cough

- WBC, ESR, and CRP normal

- Feeling of a choking sensation

- Cough worse at night; need to sleep sitting up

- Sweating episodes

- Normal between coughing episodes

morbidity as well, the isolation of the organism in 1906 led to attempts to develop vaccines for both treatment and prevention. Early studies by Madsen in the Faroe Islands in 1923-1924 and later in 1929 demonstrated some protection of vaccine recipients $[69,70]$. Early investigators in the USA were Sauer in Illinois and Kendrick and Eldering in Michigan [71, 72]. The studies done in the 1920s and 1930s were presented in depth by Lapin in 1943 [58]. I have summarized these findings before [3].

In the 1920 s and 1930 s, many different methods for vaccine preparation were used [3, 58]. It was realized that antibody responses and protection of vaccinees depended upon the number of organisms in the vaccine; it was also realized that reactions in vaccinees limited the number of organisms that could be administered in a single dose. With early vaccines, to develop protection, multiple doses of vaccine were necessary.

In 1947, Kendrick et al. developed the mouse intracerebral challenge protection test as a measure of vaccine potency [73]. This test has been used for the evaluation of whole-cell pertussis vaccine potency during the ensuing 60 years throughout the world. As will be presented later, this test allowed the use of a vaccine with substandard immunogenicity in two diphtheria and tetanus toxoids and acellular pertussis vaccine (DTaP) vaccine efficacy trials in the 1990s. Also to be noted is that an extracted vaccine (TriSolgen) was extensively used in the USA between 1962 and 1977 [3]. This vaccine (an acellular vaccine) was thought to be less reactogenic than whole-cell vaccines, but there is minimal evidence to support this view and no evidence of the vaccine's effectiveness.

\section{Acellular Vaccines (Table 5)}

During the latter half of the twentieth century, microbiologists and molecular microbiologists did extensive studies with

Table 4 History of pertussis vaccines

\begin{tabular}{ll}
\hline 1906 & B. pertussis isolated \\
$1923-1924$ & Several vaccine trials by T. Madsen; some protection \\
$1933-1942$ & Many candidate vaccines \\
1944 & Pertussis vaccine endorsed by AAP \\
1947 & Mouse protection test developed \\
1981 & Acellular vaccines used in Japan \\
\hline
\end{tabular}

Table 5 History of acellular pertussis vaccines

\begin{tabular}{|c|c|}
\hline $1930 \mathrm{~s}$ & $\begin{array}{l}\text { Many DTP vaccine preparations studied including } \\
\text { "extracted vaccines" }\end{array}$ \\
\hline 1962-1977 & An extracted vaccine (TriSolgen) available in the USA \\
\hline $1978-1981$ & DTaP vaccines developed and evaluated in Japan \\
\hline 1981 & $\begin{array}{l}\text { DTaP vaccines used routinely in Japan; starting } \\
\text { at } 2 \text { years of age }\end{array}$ \\
\hline $1980 \mathrm{~s}$ & $\begin{array}{l}\text { Many DTaP vaccines developed in North America } \\
\text { and Europe }\end{array}$ \\
\hline $1990 \mathrm{~s}$ & DTaP vaccine efficacy trials in Europe and Africa \\
\hline $1995-1997$ & $\begin{array}{l}\text { DTaP vaccines replaced DTwP vaccines in many } \\
\text { countries }\end{array}$ \\
\hline 2006 & $\begin{array}{l}\text { Tdap vaccines for adolescents and adults became } \\
\text { available }\end{array}$ \\
\hline
\end{tabular}

$D T w P$ diphtheria and tetanus toxoids and whole cell pertussis vaccine,

Tdap adolescent and adult formulated tetanus and diphtheria toxoids and acellular pertussis vaccine

B. pertussis [37]. They identified a number of proteins that were thought to be $B$. pertussis toxins or attachment factors. Most of their studies were done in mice however. The protein that gained the most attention was lymphocyte-promoting factor (LPF). Because it was thought to be the most important toxin, it was renamed "pertussis toxin" (PT). The first DTaP vaccines were developed in Japan by Sato et al. [74] It was Sato's belief that an acellular vaccine need only to contain toxoided PT. However, the first vaccines were rather impure, and in addition to PT, they contained various amounts of filamentous hemagglutinin (FHA), pertactin (PRN), and fimbriae-2 (FIM-2). In Japan, the vaccines were manufactured by six different companies. The vaccines were evaluated in small household contact studies, and aspects of these studies are presented in Table 6.

Since these studies were less than optimal, a NIH-supported controlled cohort trial was carried out in Sweden in the early 1980s [75]. This trial evaluated a single PT vaccine (without diphtheria and tetanus toxoids) with a PT/FHA-containing vaccine and determined efficacy using a placebo control group. Because there was considerable fear about possible reactions to a whole-cell vaccine, a comparative control was not included in the trial. Also, at that time in Sweden, there were some interesting opinions about the optimum age for administering pertussis vaccines; therefore, the two vaccines and the placebo were administered in two doses scheduled at $\sim 6$ and $\sim 9$ months of age. Both vaccines elicited good antibody responses to their

Table 6 Comments relating to 1980 studies in Japan

- All were household contact studies

- Most unvaccinated were $<2$ years of age and most vaccinees were $\geq 3$ years of age

- Most studies involved vaccines from more than one manufacturer

- It is likely that selection bias inflated efficacy

- All vaccines contained other proteins in addition to PT 
respective component proteins after two doses. With their primary case definition (cough illness and a positive $B$. pertussis culture), the efficacy of the PT vaccine (JNIH-7) was $54 \%$ (95\% confidence interval $(\mathrm{CI})=28-72$ ) and the $\mathrm{PT} / \mathrm{FHA}$ vaccine (JNIH-6) was $69 \%$ (95\% CI=47-82). Upon a secondary analysis which included serologic criteria JNIH-7 had no efficacy and JNIH-6 had $42 \%$ efficacy [76].

Because the studies in Japan were done in older children and the trial in Sweden was also done in older children and did not have a comparative whole-cell vaccine control, eight studies/trials involving younger infants were carried out in Europe (seven) and Africa (one). So that efficacy from different studies/trials could be compared, a case definition meeting was held by the World Health Organization on January 11, 1991 [77]. This ad hoc meeting included 22 participants, but over half of the participants were NIH, CDC, and Swedish researchers. This primary case definition is presented in Table 7. I was a member of the committee, but I strongly disagreed with the clinical criteria (more than 21 days of paroxysmal cough). We knew from our studies in Erlangen, Germany, that between 17 and $25 \%$ of unvaccinated children with pertussis would not have a paroxysmal cough lasting more than 21 days $[6,18]$.

The components of the nine vaccines used in the eight studies/trials are presented in Table 8, and the efficacy of the eight vaccines is presented in Table 9. During our trial in Erlangen, it became apparent to me that "observer bias" could inflate efficacy in all studies/trials including completely blinded trials [20]. This concept is based upon the fact that illness in controls is usually more severe than in vaccine failures. In all the studies/trials in Europe (except ours in Erlangen), the finding of cases depended upon the parents notifying the study center that their child had a cough illness. However, if the parents "knew pertussis," they would tend not to report mild cough illness. In our study in Erlangen, we kept in contact with the parents of all trial participants on an every 2-week schedule. However, it became apparent to me that only one third of our study site offices were actually doing what they were supposed to be doing. When we determined the efficacy in the offices with close monitoring, it was only $40 \%$ for the DTaP vaccine.

In the mid 1990s, a final large double-blind cohort trial without a DT control was carried out in Sweden (Sweden II) $[78,79]$. This trial involved 82,892 infants who received

Table 7 WHO pertussis case definition (Geneva January 11, 1991)

\footnotetext{
$\geq 21$ days of paroxysmal cough and one or more of the following:

- Positive culture of $B$. pertussis

- Titer rise (ELISA) IgG or IgA to PT, FHA, or FIM 2-3

- Household contact with culture confirmed case occurring \pm 28 days of onset in trial child
}

either a two-component vaccine (PT/FHA), the Italian threecomponent vaccine (PT, FHA, PRN) (Acelluvax), a Connaught (Canada) five-component vaccine (PT, FHA, PRN, FIM-2 and 3), and the Evans UK DTwP vaccine. Vaccines were administered at 3,5 , and 12 months. In determining the relative risk, the five-component vaccine was significantly better than the three-component vaccine. This five-component vaccine was later combined with $\mathrm{HiB}$ and polio. It is called Pentacel and it is routinely used in the USA. To summarize the trials of the 1990s, it can be noted that efficacy increased with the number of antigens in the vaccine and that all efficacy was inflated due to the WHO case definition and observer bias [17, 20]. Also to be noted is that the comparative DTwP vaccines in five studies/trials had greater efficacy than the comparative acellular products. The only exceptions occurred in two trials. In these two trials (Sweden I and Italy), one lot of a Connaught, USA DTwP vaccine was used, and it was later found that this vaccine had poor immunogenicity. These two trials were the hallmark NIH-supported trials. Originally, the Lederle DTwP vaccine was to be the control vaccine, but apparently NIH and Lederle could not agree on the arrangement and therefore at the last minute the Connaught USA DTwP vaccine was substituted. This vaccine had passed the mouse potency test but was, I believe, selected because of its low reactogenicity profile seen in use in the USA.

Around 2006, two adolescent/adult vaccines (Boostrix and Adacel) became available and were put into routine use [91]. The pertussis components without the diphtheria and tetanus toxoids of one of these vaccines (Boostrix) were studied in a blinded controlled trial and the efficacy was $92 \%$ [38]. Postlicensure, the effectiveness of both vaccines seemed adequate (70-80\% range). However, a recent study in Wisconsin indicated the effectiveness fell rapidly over a 3 -year period [80].

\section{Why Do DTaP and Tdap Vaccines Fail}

In 2012, I published information on this topic [49]. I will summarize this now and also in the subsequent section on the resurgence of pertussis. There are several reasons that contribute to DTaP and Tdap vaccine failure (Table 10). In children, after the third and fourth dose of DTaP, serum antibody to vaccine antigens falls rapidly [81]. However, the same is true after DTwP vaccines to the same antigens [82]. What is different is the type of cellular immune response. Using the baboon model, this has been demonstrated [65-67]. Specifically after DTwP, the cellular response is a Th1 and Th17 response, whereas following DTaP, the response is a Th1/ Th2 response. The Th1 and Th17 response allows the rapid recall of antibody in DTwP vaccine recipients, and this sustains vaccine effectiveness as was determined in the follow-up study of Sweden II [79]. 
Table 8 Characteristics of nine acellular pertussis component vaccines (DTaP vaccines)

\begin{tabular}{|c|c|c|c|c|c|c|c|}
\hline Manufacturer & $\mathrm{PT}(\mu \mathrm{g})$ & FHA $(\mu \mathrm{g})$ & PRN $(\mu \mathrm{g})$ & FIM $(\mu \mathrm{g})$ & Aluminum (mg) & Diphtheria toxoid (Lf) & Tetanus toxoid (Lf) \\
\hline Amvax & 40 & & & & 0.5 & 25 & 7 \\
\hline Connaught (US) Biken & 23.4 & 23.4 & & & 0.17 & 6.7 & 5 \\
\hline Pasteur Merieux & 25 & 25 & & & 0.3 & 15 & 5 \\
\hline SmithKline Beecham & 25 & 25 & & & 0.5 & 25 & 10 \\
\hline Chiron-Biocine & 5 & 2.5 & 2.5 & & 0.35 & 25 & 10 \\
\hline SmithKline Beecham & 25 & 25 & 8 & & 0.5 & 25 & 10 \\
\hline Connaught (Canada) & 10 & 5 & 3 & 5 & 0.33 & 15 & 5 \\
\hline Connaught (Canada) & 20 & 20 & 3 & 5 & 0.33 & 15 & 5 \\
\hline Lederle-Praxis/Takeda & 3.5 & 35 & 2 & 0.8 & 0.23 & 9 & 5 \\
\hline
\end{tabular}

All values per dose

From what was presented above, it is clear that efficacy increases directly with the number of antigens in the vaccine $[17,91]$. It should also be noted that DTwP vaccines contain $\sim 3000$ B. pertussis proteins, and antibody to many of these contribute to protection [37]. In our trial in Erlangen and in Sweden, we and they, respectively, carried out the trials in a way that serologic correlations of immunity could be determined [21, 83]. In both analyses, it was found that antibody to PRN and FIM were most important and antibody to FHA did not contribute to efficacy. We also both noted a curious finding, if you had high values to PT and FIM, you were less well protected than if you had a low value to PT. In the Swedish study but not our study, a similar relationship between PT and PRN existed. More recent studies by Weiss et al. suggest that this could be a blocking effect of excessive PT on PRN and FIM [84].
The next category, "linked epitope suppression," is more difficult to understand [42]. What our group found is that with vaccine failure, you develop antibody responses to the antigens that were in the vaccine that you had received but not to other important proteins of B. pertussis. A recent study from Australia suggests that linked epitope suppression might be very important [85]. Specifically, what they found was that children who were initially primed with DTwP vaccines had a lower attack rate than children primed with DTaP vaccines. This is particularly noteworthy because the DTwP priming occurred further back in time than the DTaP priming.

The next category (ELISA values reassured are crossreacting antibodies) relates to Tdap vaccine failures [33]. When we did the adult efficacy trial (APERT study) with the B. pertussis components of Boostrix, the decay curves to PRN and FHA were prolonged leading us to suggest that an every

Table 9 Vaccine efficacy data for eight acellular pertussis vaccines evaluated in eight trials carried out in the 1990s

\begin{tabular}{|c|c|c|c|c|c|}
\hline \multirow[t]{2}{*}{ Location } & \multirow[t]{2}{*}{ Design } & \multirow[t]{2}{*}{ Vaccine } & \multirow[t]{2}{*}{ Schedule } & \multicolumn{2}{|l|}{ Efficacy } \\
\hline & & & & $\begin{array}{l}\text { Typical } \\
\text { pertussis (\%) }\end{array}$ & $\begin{array}{l}\text { Mild and typical } \\
\text { pertussis (\%) }\end{array}$ \\
\hline Sweden, Göteborg ${ }^{\mathrm{a}}$ & Double-blind prospective cohort & Certiva & 3 doses ( 3,5 , and 12 months) & 71 & 54 \\
\hline \multirow[t]{2}{*}{ Sweden, Stockholm } & Double-blind prospective cohort & SKB-2 & 3 doses $(2,4$, and 6 months $)$ & 59 & 42 \\
\hline & & Daptacel & & 85 & 78 \\
\hline \multirow{2}{*}{ Italy, Rome } & Double-blind prospective cohort & Acelluvax & 3 doses $(2,4$, and 6 months $)$ & 84 & 71 \\
\hline & & Infanrix & & 84 & 71 \\
\hline Germany, Erlangen & Prospective cohort & Acel-Immune & 4 doses $\left(3,4 \frac{1}{2}, 6\right.$, and $15-18$ months $)$ & 83 & 72 \\
\hline Germany, Mainz & Household contact & Infanrix & 3 doses $(3,4$, and 5 months $)$ & 89 & 81 \\
\hline Germany, Munich ${ }^{\mathrm{b}}$ & Case control & Tripedia & 4 doses $(2,4,6$, and $15-25$ months $)$ & 80,93 & - \\
\hline Senegal $^{c}$ & Household contact & Triavax & 3 doses & 31,74 & - \\
\hline
\end{tabular}

Modified from Chapter 129 [91]

${ }^{a}$ Significant observer bias occurred in this trial

${ }^{\mathrm{b}}$ Laboratory diagnoses based on culture only; $80 \%$ efficacy was against cough illness of 21 or more days, and $93 \%$ efficacy was against the World Health Organization (WHO) case definition

${ }^{\mathrm{c}}$ Thirty-one percent efficacy based on 21 days or more of cough illness; $74 \%$ efficacy was against the WHO case definition 
Table 10 Possible reasons why DTaP and Tdap vaccines fail

- Decay in antibody over time

- A Th1/Th2 vs. a Th17 and Th1 response in DTaP vaccinees

- Incomplete antigen package

- Incorrect balance of antigens in the vaccine

- Linked epitope suppression

- ELISA values measured are cross reacting antibodies

- Genetic changes in B. pertussis

10-year booster vaccination schedule could control the circulation of B. pertussis in adults [33]. The recent data from Wisconsin with little efficacy after 3 years indicates we were wrong [80]. A possible explanation for this is that the antibodies that we detected in our ELISA stay elevated due to other bacteria with similar antigens, which are not effective against B. pertussis.

In the DTwP vaccine era, there was no evidence that genetic change in $B$. pertussis contributed to vaccine failure. However, with our present vaccines with three and five $B$. pertussis antigens, it is easy to see that genetic changes could be a problem. In the present era, PT has changed from ptxp1 to ptxp3. However, at present, there is no evidence that this change has contributed to vaccine failure. This is supported by the fact that in Denmark, they have been using a PT-only vaccine for 17 years and vaccine failures have not increased. More important, however, is the fact that now the majority of circulating $B$. pertussis strains are PRN-deficient mutants [86]. Since PRN is the most important antigen in Infanrix, Pediarix, and Boostrix, you would expect to see an increase failure rate with these vaccines. However, as demonstrated in Denmark, the B subunit of PT gives considerable protection against typical pertussis. Therefore, to demonstrate decreased effectiveness with these GSK vaccines, you would have to look carefully for mild illness.

\section{Epidemiology}

During the twentieth and twenty-first centuries, pertussis epidemics were and are noted to be cyclic with peaks on average of every 3 years. The common epidemiology is based upon reported pertussis and this depends upon the nature of the reporting system. Differences in rates of reported pertussis between geographic regions are mainly due to the surveillance program in a region and not due to factors of $B$. pertussis. For example, the rate of reported pertussis in Switzerland was 70fold higher than in neighboring Austria even though they had similar immunization programs [87].

During the first half of the twentieth century, 93\% of all reported cases were in children $<10$ years of age. The cycles of reported pertussis depended upon the introduction of young susceptible children into the population. Pertussis in young children was easy to recognize whereas pertussis in older people was rarely recognized as pertussis [56]. With the use of DTwP vaccines beginning in the late 1940s in the USA, the result was a 157 -fold reduction in reported pertussis by the early 1970s [2]. However, cyclic pertussis continued with the same periodicity as in the prevaccine era. Therefore, in spite of the control of pertussis by vaccination, it was apparent that B. pertussis was circulating in the population as a whole in a manner similar to the prevaccine era [4]. If you look at measles control through vaccination, you see a decreased number of cases, but more importantly, you see a lengthening of the inter-epidemic cycle. Since this has not happened with pertussis, we know that $B$. pertussis is circulating in a fashion similar to that which occurred in the prevaccine era. This is due to the knowledge that immunity following natural infection on immunization is short lived and therefore reinfection illnesses are common [11, 37, 91]. Until relatively recently, virtually all adolescent and adult cases were misdiagnosed. And even today, most adult cases are initially misdiagnosed. In the 1930s, many physicians with an interest in pertussis recognized adult pertussis but underestimated its prevalence [58, 62].

In contrast with the analysis of reported pertussis in different age groups, there has been considerable progress in studying $B$. pertussis infections in adolescents and adults [36]. This has involved these approaches (1) studying patients with prolonged cough illnesses, (2) looking at serum B. pertussis antibodies in specific individuals sequentially, and (3) studying the rate of $B$. pertussis cough illness in defined populations.

Since adolescents and adults usually do not seek medical care until the third or fourth week of illness, they most often will not have a positive culture or also a positive PCR assay [4, 12]. Therefore, most studies of adolescent and adult cough illnesses have employed serologic study with ELISA. Again, since most cases are not seen early in their illnesses, antibody titer rises between acute- and convalescent-phase specimens are unlikely to show significant increases in values. Therefore, what has been used is the demonstration of high values in single-serum specimens using validated cutoff points that were determined. In many of the original studies, antibody values to PT, FHA, PRN, FIM, and agglutinogens were determined. However, since antibody to all antigens except PT may be due to other Bordetella sp. and other microorganisms as well, B. pertussis cough illnesses were overestimated. Therefore, the use of antibody to just PT is the test of choice. With this criterion, it appears that about $13 \%$ of prolonged afebrile cough illness are due to $B$. pertussis infections [36].

In the 5-year period between 1985 and 1989, we had yearly sera from 53 health care workers [13]. When we looked at significant ELISA antibody titer rises to PT, we found that $\sim 6 \%$ per year had B. pertussis infections. During subsequent years, similar studies were done by other investigations and all 
found high rates of infection (between 1 and $3 \%$ per year) but with one exception, these studies had sera at just two points in time. The exception was a study that we did in Cleveland in persons $>65$ years of age who were living in the community. In this study, we had sera collected every 4 months for 3 years. The infection rate was $3 \%$ per year. More recently in the Netherlands, using a different methodology, they confirmed our original $6 \%$ rate and also our $3 \%$ rate in those $>65$ years of age [88]. Of course in all of these investigations (except our Cleveland study), these all indicate rates of infection, and they tell us nothing about illness rates.

Attempts to determine illness rates were made in three of the original cough illness studies since knowledge of the overall populations (denominator) were thought to be known. The following rates were determined: UCLA students 69 per 100 , 000 per year, Northern California Kaiser 176 per 100,000 per year, and adults in Germany 133 per 100,000 per year [4, 12, 89]. The first prospective study was done in Minnesota and the rate was 500 per 100,000 [90]. However, this rate is an underestimate because there was considerable observer bias by the study physicians and therefore many mild cases may have been missed. The second study involved the $\sim 1400$ controls in our adolescent/adult efficacy trial (APERT trial) [38, 40]. The rate here was 370 per 100,000. This also is an underestimate because of observed bias by the study nurses. The study done in adults $>65$ years of age in Cleveland was originally a respiratory viral/Mycoplasma pneumoniae study and clinical information was available. If all the cough illnesses in the 4-month periods with significant PT titer rises are considered as cases, the rate could have been as high as $1.5 \%$ [28].

Collectively, these three types of $B$. pertussis infection studies indicate the following: (1) B. pertussis cough illnesses are endemic in adolescents and adults, (2) pertussis surveillance only finds the tip of the iceberg in regard to actual cases, and (3) asymptomatic infections are 4-20 times more common than symptomatic cases.

\section{Pathophysiology/Pathology}

\section{Pathology}

The only available data on the pathology of $B$. pertussis infections in humans comes from postmortem studies of pertussis-related deaths $[41 \bullet \bullet, 55 \bullet \cdot, 60 \bullet \cdot$. Data from these studies is often compromised by concomitant and secondary bacterial or viral infections. Nevertheless, the findings of Mallory and Horner over 100 years ago are revealing [60••]. They noted that the ciliated cells of the trachea, bronchi, and bronchioles were largely intact. The cilia themselves were covered with bacteria. In 2008, Paddock et al. noted similar findings [55••]. In addition, our findings in the specimens from 15 young infant deaths noted that the lumens of arterioles, venules, and lymphatics were clogged by aggregates of mature neutrophils and lymphocytes. These cells reflected the leukocytosis with lymphocytosis in the blood of these patients prior to death. Although concomitant or secondary bacterial or viral infections made conclusions about the histopathology in individual cases difficult, it is my opinion that necrosis in the alveoli (as would occur in viral infections) or an extensive neutrophil inflammatory response (with band forms) as would be seen in bacterial pneumonia is not a pulmonary manifestation of $B$. pertussis infection. Pulmonary macrophages were present in interstitial spaces and in the alveoli.

The histopathological findings in the lungs of the infant pertussis deaths is completely different from that in young mice with $B$. pertussis infection (personal observation by Paddock and the author).

\section{Pathophysiology}

As noted previously, over the last 100 years, numerous toxins and biologically active proteins have been isolated and studied in animal model systems (mainly in mice) [37, 53, 55••]. Suggested toxins are PT, DNT, adenylate cyclase toxin (ACT), endotoxin (LPS), and tracheal cytotoxin (TCT). In addition, other proteins were thought to be important as adhesins, these include FIM, PRN and other autotransporters, and FHA. These studies led directly to the formulation of acellular pertussis vaccines.

Now it is apparent that many of the ideas put forward in the 1970s were wrong. For example, the human pathology suggests that neither DNT nor TCT have a role in human infection. There is also little evidence that ACT plays any role in human infection although it is immunogenic and it elicits a strong antibody response [30]. LPS is also highly immunogenic but its adverse effects (fever, shock) are not observed in human infections. PRN and similar proteins (other autotransporters) are not adhesins but are in fact toxins that adversely affect the innate immune response. The only adhesins are fimbriae 2 and $3[53,55 \bullet \cdot]$.

Therefore, it is apparent that multiple toxins and one adhesin all have roles in human $B$. pertussis infections. In contrasts, however, there are only two toxins that cause clinical illness. One of these is PT and the other is the toxin that causes the cough. Our group has described the mechanism of illnesses caused by PT [51••]. This illness is related to the leukocytosis with lymphocytosis and is the cause of deaths in young infants. Once a person has been vaccinated or has had pertussis, they never again have symptoms that can be attributed to PT. For example, leukocytosis with lymphocytosis does not occur in adult illnesses.

In contrast with PT, nothing is known about the cough toxin [55••]. All persons with pertussis (both primary and repeat infections) will have the distinctive paroxysm cough. 
This suggests to me that the cough toxin is not immunogenic in natural infectious and not immunogenic in or a component of whole-cell vaccines.

\section{The Resurgence of Reported Pertussis}

Many of the prevailing opinions relating to the "resurgence of pertussis" are incomplete or are wrong. There are four misconceptions relating to the "resurgence" of pertussis. The first of these is that it is due to the inferiority of DTaP vaccines compared with DTwP vaccines. Although the DTaP vaccines are contributing to the problem, it is important to point out that the resurgence had its onset $\sim 14$ years before DTaP vaccines replaced DTwP vaccines in the USA. The second misconception is that genetic changes in B. pertussis are the cause of the resurgence. Although I think that PRN-deficient mutants are contributing to DTaP vaccine failure, the resurgence had its onset before this mutation occurred. There has also been a mutation in PT (ptxp1 to ptxp3) but there is no evidence that this has contributed to vaccine failure. Since in Denmark they have used a PT vaccine without other antigens for 17 years, you would expect major epidemics in that country and that is not the case. A third misconception is that immunity following pertussis is life-long whereas immunity following immunization with either DTwP or DTaP is relatively shortlived. In studies that our groups in Germany and the USA did, the findings indicate that immunity following DTwP immunization was actually better than that following natural infection [11].

The fourth misconception is that pertussis in adolescents and adults is a new phenomenon due to changes in herd immunity in the vaccine area. This is also incorrect in that adult pertussis was occurring in the prevaccine era in a fashion similar to the present era.

The fact today is that when you look at endemic and epidemic disease combined, it is clear that reported pertussis today is $\sim 20$-fold less than what it was in the prevaccine era. In addition, illness in DTaP vaccine failures is less severe than illness in similar-aged unvaccinated children.

It is clear that the most important factor in the resurgence of pertussis is increased awareness. In the 1980s, hundreds of papers were published about DTaP vaccine reactions at the development of DTaP vaccines. Following this, there were hundreds of papers relating to the testing of DTaP vaccines in the 1990s. Most recently, hundreds of communications have related to lack of DTaP vaccine efficiency.

The second most important factor relating to the resurgence is the use of PCR for diagnosis. PCR sensitivity uncovers many more cases than conventional culture. It should also be noted that if single-serum diagnosis by determining significant values of antibody to PT was to be used more routinely, the number of confirmed adolescent and adult cases would be considerably greater. In fact, if this was routinely used in adults of all ages, the pertussis cycles would disappear.

Other contributors to DTaP and Tdap vaccine failures in the resurgence of pertussis include decay in antibody over time, a Th1/Th2 vs. a Th1 and Th17 cellular response in DTaP vaccines, incomplete antigen package, incorrect balance of antigens in the vaccine, linked epitope suppression, ELISA values measured are cross-reacting antibodies (these are specific for Tdap vaccine failures), and genetic changes in B. pertussis.

\section{Summary/Conclusions}

Of all respiratory illness infections, pertussis is unique. B. pertussis cough illness occurs without inflammation unless there is a concomitant or secondary bacterial or viral infection. Over the last 100 years, pertussis and $B$. pertussis have been extensively studied. The use of animal model systems led to the finding of many $B$. pertussis virulence factors. However, much of what was learned was wrong, and good laboratory and observational studies have been overlooked.

In spite of the fact that the resurgence of pertussis has largely been interpreted incorrectly, it is clear that a major priority today should be the development of new vaccines. However, the logistical problems relating to the development and testing of new vaccines are huge. Since placebocontrolled trials today would not be ethical, the sample sizes to determine efficacy would be cost prohibitive. Therefore, surrogate markers would need to br developed that would be acceptable by regulatory authorities.

There would seem to be two approaches to the development of new pertussis vaccines. One of these would be a new acellular pertussis vaccine. This would require a method that would ensure a Th1 and Th17 cellular response. Secondly, relating to the possibility of genetic mutations (never a problem with DTwP vaccines) would be making a decision as to how many additional antigens would be necessary so that mutation would not be a factor. The third consideration would be the balance of the concentration of the antigens included in the new vaccine. For example, studies done almost 20 years ago suggest that FHA should not be included in a new formulation. In addition, although PT is a critical vaccine component, too much may be detrimental.

Another approach would be to make a new DTwP vaccine with the inflammatory Toll-like receptor (TLR)-4 response to LPS-attenuated. In my opinion, this approach is possibly simpler than the development of a new DTaP vaccine. LPS has many important properties which are in present DTwP vaccines and it is important that these are retained and only the TLR-4 inflammatory response be attenuated.

Finally, we should use our present vaccines in the best possible way. In many countries, DTwP vaccines are still used and their continued use should be supported. What should be 
encouraged in these countries is the use of Tdap in pregnant women as this can prevent most pertussis-related deaths in young infants $[54,92]$.

In the USA and other countries using DTaP and Tdap vaccines, we should concentrate on preventing severe and fatal pertussis. This can be achieved by Tdap vaccination in all pregnant women and giving the first dose of DTaP at 6 weeks of age rather than at 2 months of age [93].

\section{Compliance with Ethics Guidelines}

Conflict of Interest JD Cherry has received a speaker honorarium from Sanofi Pasteur and is a member of GPI (supported by Sanofi Pasteur).

Human and Animal Rights and Informed Consent All studies by James Cherry involving animal and/or human subjects were performed after approval by the appropriate institutional review boards. When required, written informed consent was obtained from all participants.

\section{References}

Papers of particular interest, published recently, have been highlighted as:

•- Of major importance

1. Cody CL, Baraff LJ, Cherry JD, Marcy SM, Manclark CR. Nature and rates of adverse reactions associated with DTP and DT immunizations in infants and children. Pediatrics. 1981;68:650-60.

2. Cherry JD. The epidemiology of pertussis and pertussis immunization in the United Kingdom and the United States: a comparative study. Curr Probl Pediatr. 1984;14:1-78.

3. Cherry JD, Brunell PA, Golden GS, Karzon DT. Report of the task force on pertussis and pertussis immunization-1988. Pediatrics. 1988;81:939-84.

4. Mink CM, Cherry JD, Christenson P, et al. A search for Bordetella pertussis infection in university students. Clin Infect Dis. 1992;14: 464-71.

5. Cherry JD. Pertussis vaccine encephalopathy: it is time to recognize it as the myth that it is. JAMA. 1990;263:1679-80.

6. Heininger U, Cherry JD, Eckhardt T, Lorenz C, Christenson P, Stehr K. Clinical and laboratory diagnosis of pertussis in the regions of a large vaccine efficacy trial in Germany. Pediatr Infect Dis J. 1993; 12:504-9.

7. Beiter A, Lewis K, Pineda EF, Cherry JD. Unrecognized maternal peripartum pertussis with subsequent fatal neonatal pertussis. Obstet Gynecol. 1993;82:691-3.

8. Heininger U, Cherry JD, Christenson PD, et al. Comparative study of Lederle/Takeda acellular and Lederle whole-cell pertussis-component diphtheria-tetanus-pertussis vaccines in infants in Germany. Vaccine. 1994;12:81-6.

9. Cherry JD, Olin P. The science and fiction of pertussis vaccines. Pediatrics. 1999;104:1381-3.

10. Cherry JD. The science and fiction of the "resurgence" of pertussis. Pediatrics. 2003;112:405-6.

11. Cherry JD, Beer T, Chartrand SA, et al. Comparison of values of antibody to Bordetella pertussis antigens in young German and American men. Clin Infect Dis. 1995;20:1271-4.
12. Schmitt-Grohe S, Cherry JD, Heininger U, Uberall MA, Pineda E, Stehr K. Pertussis in German adults. Clin Infect Dis. 1995;21: 860-6.

13. Deville JG, Cherry JD, Christenson PD, et al. Frequency of unrecognized Bordetella pertussis infections in adults. Clin Infect Dis. 1995;21:639-42.

14. Deen JL, Mink CA, Cherry JD, et al. Household contact study of Bordetella pertussis infections. Clin Infect Dis. 1995;21:1211-9.

15. Heininger U, Stehr K, Schmidt-Schlapfer G, et al. Bordetella pertussis infections and sudden unexpected deaths in children. Eur $\mathbf{J}$ Pediatr. 1996;155:551-3.

16. Cherry JD. The role of Bordetella pertussis infections in adults in the epidemiology of pertussis. Dev Biol Stand. 1997;89:181-6.

17. Cherry JD. Comparative efficacy of acellular pertussis vaccines: an analysis of recent trials. Pediatr Infect Dis J. 1997;16:S90-6.

18. Heininger U, Klich K, Stehr K, Cherry JD. Clinical findings in Bordetella pertussis infections: results of a prospective multicenter surveillance study. Pediatrics. 1997;100:E10.

19. Stehr K, Cherry JD, Heininger U, et al. A comparative efficacy trial in Germany in infants who received either the Lederle/Takeda acellular pertussis component DTP (DTaP) vaccine, the Lederle wholecell component DTP vaccine, or DT vaccine. Pediatrics. 1998;101: $1-11$.

20. Cherry JD, Heininger U, Stehr K, Christenson P. The effect of investigator compliance (observer bias) on calculated efficacy in a pertussis vaccine trial. Pediatrics. 1998;102:909-12.

21. Cherry JD, Gornbein J, Heininger U, Stehr K. A search for serologic correlates of immunity to Bordetella pertussis cough illnesses. Vaccine. 1998;16:1901-6.

22. Heininger U, Cherry JD, Stehr K, et al. Comparative Efficacy of the Lederle/Takeda acellular pertussis component DTP (DTaP) vaccine and Lederle whole-cell component DTP vaccine in German children after household exposure. Pertussis Vaccine Study Group. Pediatrics. 1998;102:546-53.

23. Cherry JD. Pertussis in the preantibiotic and prevaccine era, with emphasis on adult pertussis. Clin Infect Dis. 1999;28 Suppl 2: S107-11.

24. Cherry JD. Epidemiological, clinical, and laboratory aspects of pertussis in adults. Clin Infect Dis. 1999;28 Suppl 2:S112-7.

25. Heininger U, Schmidt-Schlapfer G, Cherry JD, Stehr K. Clinical validation of a polymerase chain reaction assay for the diagnosis of pertussis by comparison with serology, culture, and symptoms during a large pertussis vaccine efficacy trial. Pediatrics. 2000;105: E31.

26. Vincent JM, Cherry JD, Nauschuetz WF, et al. Prolonged afebrile nonproductive cough illnesses in American soldiers in Korea: a serological search for causation. Clin Infect Dis. 2000;30:534-9.

27. Jackson LA, Cherry JD, Wang SP, Grayston JT. Frequency of serological evidence of Bordetella infections and mixed infections with other respiratory pathogens in university students with cough illnesses. Clin Infect Dis. 2000;31:3-6.

28. Hodder SL, Cherry JD, Mortimer Jr EA, Ford AB, Gornbein J, Papp K. Antibody responses to Bordetella pertussis antigens and clinical correlations in elderly community residents. Clin Infect Dis. 2000;31:7-14.

29. Lugauer S, Heininger U, Cherry JD, Stehr K. Long-term clinical effectiveness of an acellular pertussis component vaccine and a whole cell pertussis component vaccine. Eur J Pediatr. 2002;161: $142-6$.

30. Cherry JD, Xing DX, Newland P, Patel K, Heininger U, Corbel MJ. Determination of serum antibody to Bordetella pertussis adenylate cyclase toxin in vaccinated and unvaccinated children and in children and adults with pertussis. Clin Infect Dis. 2004;38:502-7.

31. Heininger U, Cherry JD, Stehr K. Serologic response and antibodytiter decay in adults with pertussis. Clin Infect Dis. 2004;38:591-4. 
32. Heininger U, Kleemann WJ, Cherry JD, Sudden Infant Death Syndrome Study Group. A controlled study of the relationship between Bordetella pertussis infections and sudden unexpected deaths among German infants. Pediatrics. 2004;114:e9-15.

33. Le T, Cherry JD, Chang SJ, et al. Immune responses and antibody decay after immunization of adolescents and adults with an acellular pertussis vaccine: the APERT Study. J Infect Dis. 2004;190:535-44.

34. Cherry JD, Chang SJ, Klein D, et al. Prevalence of antibody to Bordetella pertussis antigens in serum specimens obtained from 1793 adolescents and adults. Clin Infect Dis. 2004;39:1715-8.

35. Cherry JD, Grimprel E, Guiso N, Heininger U, Mertsola J. Defining pertussis epidemiology: clinical, microbiologic and serologic perspectives. Pediatr Infect Dis J. 2005;24:S25-34.

36. Cherry JD. The epidemiology of pertussis: a comparison of the epidemiology of the disease pertussis with the epidemiology of Bordetella pertussis infection. Pediatrics. 2005;115:1422-7.

37. Mattoo S, Cherry JD. Molecular pathogenesis, epidemiology, and clinical manifestations of respiratory infections due to Bordetella pertussis and other Bordetella subspecies. Clin Microbiol Rev. 2005; 18:326-82.

38. Ward JI, Cherry JD, Chang SJ, et al. Efficacy of an acellular pertussis vaccine among adolescents and adults. N Engl $\mathrm{J}$ Med. 2005;353:1555-63.

39. Cherry JD. Immunity to pertussis. Clin Infect Dis. 2007;44:1278-9.

40. Ward JI, Cherry JD, Chang SJ, et al. Bordetella pertussis infections in vaccinated and unvaccinated adolescents and adults, as assessed in a national prospective randomized Acellular Pertussis Vaccine Trial (APERT). Clin Infect Dis. 2006;43:151-7.

41.• Paddock CD, Sanden GN, Cherry JD, et al. Pathology and pathogenesis of fatal Bordetella pertussis infection in infants. Clin Infect Dis. 2008;47:328-38. This is the most complete study to date on hist. pathologic findings in fatal pertussis.

42. Cherry JD, Heininger U, Richards DM, et al. Antibody response patterns to Bordetella pertussis antigens in vaccinated (primed) and unvaccinated (unprimed) young children with pertussis. Clin Vaccine Immunol. 2010;17:741-7.

43. Cherry JD. The present and future control of pertussis. Clin Infect Dis. 2010;51:663-7.

44. Cherry JD, Seaton BL. Patterns of Bordetella parapertussis respiratory illnesses: 2008-2010. Clin Infect Dis. 2012;54:534-7.

45. Prince HE, Lieberman JM, Cherry JD. Age-related differences in patterns of increased Bordetella pertussis antibodies. Clin Vaccine Immunol. 2012;19:545-50.

46. Cherry JD. The protein content of diphtheria-tetanus toxoids-acellular pertussis vaccines and an emerging clinical problem. J Infect Dis. 2005;191:1386-8.

47. Cherry JD. Historical perspective on pertussis and use of vaccines to prevent it. Microbe. 2007;2:139-44.

48. Cherry JD, Tan T, Wirsing von Konig CH, et al. Clinical definitions of pertussis: summary of a Global Pertussis Initiative roundtable meeting, February 2011. Clin Infect Dis. 2012;54:1756-64.

49. Cherry JD. Why do pertussis vaccines fail? Pediatrics. 2012;129: 968-70.

50. Cherry JD. Epidemic pertussis in 2012 - the resurgence of a vaccine-preventable disease. N Engl J Med. 2012;367:785-7.

51.• Murray EL, Nieves D, Bradley JS, et al. Characteristics of severe Bordetella pertussis infection among infants $<90$ days of age admitted to pediatric intensive care units-Southern California, September 2009-June 2011. J Pediatr Infect Dis Soc. 2013;2:1-6. This is the most complete study to date on severe pertussis in young infants. It demonstrates four factors relating to fatal pertussis/pulmonary hypertension.

52. Nieves D, Bradley JS, Gargas J, et al. Exchange blood transfusion in the management of severe pertussis in young infants. Pediatr Infect Dis J. 2013;32:698-9.
53. Cherry JD. Pertussis: challenges today and for the future. PLoS Pathog. 2013;9:e1003418.

54. Cherry JD. Editorial commentary: tetanus-diphtheria-pertussis immunization in pregnant women and the prevention of pertussis in young infants. Clin Infect Dis. 2015;60:338-40.

55.• Cherry JD, Paddock CD. Pathogenesis and histopathology of pertussis: implications for immunization. Expert Rev Vaccines. 2014; 13:1115-23. This is the most complete review of pathogenesis and hist. pathology of pertussis.

56. Cherry JD. Adult pertussis in the pre- and post-vaccine eras: lifelong vaccine-induced immunity? Expert Rev Vaccines. 2014;13: 1073-80.

$57 . \bullet$ Holmes WH. Bacillary and rickettsial infections: acute and chronic: a textbook. Black death to white plague. New York: Macmillan; 1940. Holmes pointed out the unique features of pertussis which are often overlooked today.

58. Lapin JH. Whooping cough. Springfield: Charles Thomas; 1943.

59. Bordet J, Gengou O. Le microbe de la coqueluche. Ann Inst Pasteur (Paris). 1906;20:48-68.

60.• Mallory FB, Hornor AA. Pertussis: the histological lesion in the respiratory tract. J Med Res. 1912;27:115-243. This study noted relatively normal ciliated respiratory epithelial cells in fatal pertussis cases.

61. Holt LE. The diseases of infancy and childhood: for the use of students and practitioners in medicine. New York: D. Appleton; 1902.

62. Mannerstedt G. Pertussis in adults. J Pediatr. 1934;5:596-600.

63. Bordet J, Gengou O. L'endotoxin coqueluchese. Ann Inst Pasteur (Paris). 1909;23:415-9.

64. Parkhill J, Sebaihia M, Preston A, et al. Comparative analysis of the genome sequences of Bordetella pertussis, Bordetella parapertussis and Bordetella bronchiseptica. Nat Genet. 2003;35:32-40.

65. Warfel JM, Merkel TJ. Bordetella pertussis infection induces a mucosal IL-17 response and long-lived Th17 and Th1 immune memory cells in nonhuman primates. Mucosal Immunol. 2013;6:78796.

66. Warfel JM, Papin JF, Wolf RF, Zimmerman LI, Merkel TJ. Maternal and neonatal vaccination protects newborn baboons from pertussis infection. J Infect Dis. 2014;210:604-10.

67. Warfel JM, Zimmerman LI, Merkel TJ. Acellular pertussis vaccines protect against disease but fail to prevent infection and transmission in a nonhuman primate model. Proc Natl Acad Sci U S A. 2014;111:787-92.

68. Taylor ZW, Ackerson B, Bronstein DE, et al. Wheezing in children with pertussis associated with delayed pertussis diagnosis. Pediatr Infect Dis J. 2014;33:351-4.

69. Madsen T. Whooping cough: its bacteriology, diagnosis, prevention and treatment. Boston Med Surg J. 1925;192:50-60.

70. Madsen T. Vaccination against whooping cough. JAMA. 1933;101: $187-8$.

71. Sauer LW, Tucker WH. Simultaneous administration of diphtheria toxoid and pertussis vaccine in young children. Am J Public Health Nations Health. 1942;32:385-8.

72. Kendrick P, Eldering G. A study in active immunization against pertussis. Am J Hyg. 1939;29:133-53.

73. Kendrick PL, Eldering G, et al. Mouse protection tests in the study of pertussis vaccine; a comparative series using the intracerebral route for challenge. Am J Public Health Nations Health. 1947;37: 803-10.

74. Sato Y, Kimura M, Fukumi H. Development of a pertussis component vaccine in Japan. Lancet. 1984;1:122-6.

75. Ad Hoc Group for the Study of Pertussis Vaccines. Placebocontrolled trial of two acellular pertussis vaccines in Swedenprotective efficacy and adverse events. Lancet. 1988;1:955-60.

76. Storsaeter J, Hallander H, Farrington CP, Olin P, Mollby R, Miller E. Secondary analyses of the efficacy of two acellular pertussis 
vaccines evaluated in a Swedish phase III trial. Vaccine. 1990;8: 457-61.

77. World Health Organization. WHO meeting on case definition of pertussis: Geneva 10-11 January 1991. Geneva: Switzerland; 1991.

78. Olin P, Rasmussen F, Gustafsson L, Hallander HO, Heijbel H. Randomised controlled trial of two-component, three-component, and five-component acellular pertussis vaccines compared with whole-cell pertussis vaccine. Ad Hoc Group for the Study of Pertussis Vaccines. Lancet. 1997;350:1569-77.

79. Gustafsson L, Hessel L, Storsaeter J, Olin P. Long-term follow-up of Swedish children vaccinated with acellular pertussis vaccines at 3,5 , and 12 months of age indicates the need for a booster dose at 5 to 7 years of age. Pediatrics. 2006;118:978-84.

80. Koepke R, Eickhoff JC, Ayele RA, et al. Estimating the effectiveness of tetanus-diphtheria-acellular pertussis vaccine (Tdap) for preventing pertussis: evidence of rapidly waning immunity and difference in effectiveness by Tdap brand. J Infect Dis. 2014;210: 942-53.

81. Guerra FA, Blatter MM, Greenberg DP, Pichichero M, Noriega FR, Pentacel Study G. Safety and immunogenicity of a pentavalent vaccine compared with separate administration of licensed equivalent vaccines in US infants and toddlers and persistence of antibodies before a preschool booster dose: a randomized, clinical trial. Pediatrics. 2009;123:301-12.

82. Blumberg DA, Mink CM, Cherry JD, et al. Comparison of an acellular pertussis-component diphtheria-tetanus-pertussis (DTP) vaccine with a whole-cell pertussis-component DTP vaccine in 17- to 24-month-old children, with measurement of 69-kilodalton outer membrane protein antibody. J Pediatr. 1990;117:46-51.

83. Storsaeter J, Hallander HO, Gustafsson L, Olin P. Levels of antipertussis antibodies related to protection after household exposure to Bordetella pertussis. Vaccine. 1998;16:1907-16.
84. Weiss AA, Patton AK, Millen SH, Chang SJ, Ward JI, Bernstein DI. Acellular pertussis vaccines and complement killing of Bordetella pertussis. Infect Immun. 2004;72:7346-51.

85. Sheridan SL, Ware RS, Grimwood K, Lambert SB. Number and order of whole cell pertussis vaccines in infancy and disease protection. JAMA. 2012;308:454-6.

86. Pawloski LC, Queenan AM, Cassiday PK, et al. Prevalence and molecular characterization of pertactin-deficient Bordetella pertussis in the United States. Clin Vaccine Immunol. 2014;21:119-25.

87. Celentano LP, Massari M, Paramatti D, Salmaso S, Tozzi AE, Group E-N. Resurgence of pertussis in Europe. Pediatr Infect Dis J. 2005;24:761-5.

88. de Melker HE, Versteegh FG, Schellekens JF, Teunis PF, Kretzschmar M. The incidence of Bordetella pertussis infections estimated in the population from a combination of serological surveys. J Infect. 2006;53:106-13.

89. Nennig ME, Shinefield HR, Edwards KM, Black SB, Fireman BH. Prevalence and incidence of adult pertussis in an urban population. JAMA. 1996;275:1672-4.

90. Strebel P, Nordin J, Edwards K, et al. Population-based incidence of pertussis among adolescents and adults, Minnesota, 1995-1996. J Infect Dis. 2001;183:1353-9.

91. Cherry JD, Heininger U. Pertussis and other Bordetella infections. In: Cherry JD, Harrison GJ, Kaplan SL, Steinbach WJ, Hotez PJ, editors. Feigin and Cherry's textbook of pediatric infectious diseases. 7th ed. Philadelphia: Elsevier Saunders; 2014. p. 1616-39.

92. Dabrera G, Amirthalingam G, Andrews N, et al. A case-control study to estimate the effectiveness of maternal pertussis vaccination in protecting newborn infants in England and Wales, 2012-2013. Clin Infect Dis. 2015;60:333-7.

93. Shinall Jr MC, Peters TR, Zhu Y, Chen Q, Poehling KA. Potential impact of acceleration of the pertussis vaccine primary series for infants. Pediatrics. 2008;122:1021-6. 